\title{
BOUNDARY VALUE PROBLEMS FOR HOLOMORPHIC FUNCTIONS ON THE UPPER HALF-PLANE*
}

\author{
MIRAN ČERNE ${ }^{\dagger}$ AND MANUEL FLORES $\ddagger$
}

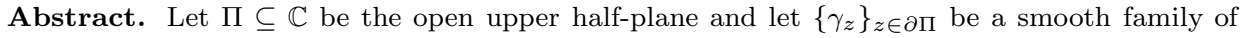
smooth Jordan curves in the complex plane $\mathbb{C}$ parametrized by the boundary of $\Pi$. Then there exists a smooth up to the boundary holomorphic function $f$ on $\Pi$ such that $f(z) \in \gamma_{z}$ for every $z \in \partial \Pi$. Similar result is also proved on an arbitrary bordered Riemann surface.
\end{abstract}

Key words. Boundary value problem, Riemann-Hilbert problem

AMS subject classifications. Primary 30E25, 35Q15

1. Introduction. Let $\Pi=\{z \in \mathbb{C} ; \operatorname{Im}(\mathrm{z})>0\}$ be the open upper half-plane and let $\left\{\gamma_{z}\right\}_{z \in \partial \Pi}$ be a smooth family of smooth Jordan curves in the complex plane parametrized by the boundary $\partial \Pi$ of $\Pi$, that is, there exists a function $\rho \in C^{\infty}(\partial \Pi \times \mathbb{C})$ such that

$$
\gamma_{z}=\{w \in \mathbb{C} ; \rho(z, w)=0\}
$$

and $\bar{\partial}_{w} \rho(z, w) \neq 0$ for every $z \in \partial \Pi$ and $w \in \gamma_{z}$. We are interested in the existence of solutions of the corresponding Riemann-Hilbert problem and we show the following theorem.

TheOREM 1.1. Let $\left\{\gamma_{z}\right\}_{z \in \partial \Pi}$ be a smooth family of smooth Jordan curves in $\mathbb{C}$. Then there exists a smooth up to the boundary holomorphic function $f$ on $\Pi$ such that $f(z) \in \gamma_{z}$ for every $z \in \partial \Pi$.

Using conformal equivalence between the open upper half-plane $\Pi$ and the open unit disc $\Delta$ one gets the following equivalent statement.

ThEOREM 1.2. Let $\left\{\gamma_{z}\right\}_{z \in \partial \Delta \backslash\{1\}}$ be a smooth family of smooth Jordan curves in $\mathbb{C}$. Then there exists a smooth function $f$ on $\bar{\Delta} \backslash\{1\}$, holomorphic on $\Delta$, such that $f(z) \in \gamma_{z}$ for every $z \in \partial \Delta \backslash\{1\}$.

Let $\left\{\gamma_{z}\right\}_{z \in \partial \Delta}$ be a smooth family of smooth Jordan curves in $\mathbb{C}$ parametrized by the whole boundary $\partial \Delta$ of $\Delta$. By Theorem 1.2 there are no obstructions to the existence of a solution of the Riemann-Hilbert problem on the disc for the family of Jordan curves $\left\{\gamma_{z}\right\}_{z \in \partial \Delta}$ if we allow solutions to be "wild" at only one boundary point. On the other hand the existence of a smooth up to the boundary holomorphic function $f$ on $\Delta$ such that $f(z) \in \gamma_{z}$ for every $z \in \partial \Delta$ is not always guaranteed. For example one can take

$$
\rho(z, w)=|w-\bar{z}|^{2}-r^{2},
$$

\footnotetext{
*Received August 28, 2006; accepted for publication September 8, 2006.

${ }^{\dagger}$ Department of Mathematics, University of Ljubljana, Jadranska 19, 1111 Ljubljana, Slovenia (miran.cerne@fmf.uni-lj.si). The first author was supported in part by a grant Analiza in geometrija P1-0291 from the Ministry of Higher Education, Science and Technology of the Republic of Slovenia.

$\ddagger$ Department of Mathematics, University of La Laguna, 38771 La Laguna, Tenerife, Spain (mflores @ull.es). The second author was supported in part by grants from FEDER y Ministerio de Ciencia y Tecnologia number MTM 2004-05878 and Consejeria de Educacion Cultura y Deportes del Gobierno de Canarias, PI 2003/068.
} 
that is, $\gamma_{z}$ is a circle with radius $r$ centered at $\bar{z}$. It is easy to see, using either the maximum principle or the argument principle, that in the case $0<r<1$ there is no holomorphic function $f$ on $\Delta$ which is continuous on $\bar{\Delta}$ and such that $f(z) \in \gamma_{z}$ for every $z \in \partial \Delta$. Forstnerič [13] and independently Šnirelman [23] showed that the assumption on the existence of an analytic selector for the family $\left\{\gamma_{z}\right\}_{z \in \partial \Delta}$ is sufficient for the existence of a smooth up to the whole boundary solution of the corresponding Riemann-Hilbert problem. A holomorphic function $g$ on $\Delta$ which is continuous on $\bar{\Delta}$ is said to be an analytic selector for the family of Jordan curves $\left\{\gamma_{z}\right\}_{z \in \partial \Delta}$ if $g(z)$ belongs to the bounded component of $\mathbb{C} \backslash \gamma_{z}$ for every $z \in \partial \Delta$. Later Slodkowski [22] proved that there exists a solution of the Riemann-Hilbert problem for $\left\{\gamma_{z}\right\}_{z \in \partial \Delta}$ if and only if the polynomial hull of the maximal real torus

$$
T=\cup_{z \in \partial \Delta}\left(\{z\} \times \gamma_{z}\right) \subseteq \partial \Delta \times \mathbb{C}
$$

is nontrivial, that is, it contains a point over $\Delta$. See also [25].

On the formal level Theorem 1.2 seems to be similar to a result by Alexander [1] on the existence of a nontrivial nearly smooth analytic disc with boundary in a given maximal real submanifold. However, there is a major difference between these two results. All analytic discs considered by Alexander in [1] are bounded which is in general far from being the case in Theorem 1.2.

It also follows from $[13,22,23]$ that the family of smooth up to the whole boundary solutions of the Riemann-Hilbert problem on the disc is quite "rigid": it is the union of at most countably many finite dimensional manifolds. If the regularity of solutions of the Riemann-Hilbert problem is not required at only one boundary point, we have, as it should be expected, much more flexibility. In addition to Theorem 1.1 we also have a version with approximation.

THEOREM 1.3. Let $\left\{\gamma_{z}\right\}_{z \in \partial \Pi}$ be a smooth family of smooth Jordan curves in $\mathbb{C}$. Let $\Gamma: \partial \Pi \rightarrow \mathbb{C}$ be a $C^{k}(k \in \mathbb{N} \cup\{0\})$ function such that $\Gamma(z) \in \gamma_{z}$ for every $z \in \partial \Pi$ and let $\varepsilon: \partial \Pi \rightarrow(0, \infty)$ be a continuous positive function. Then there exists a smooth up to the boundary holomorphic function $f$ on $\Pi$ such that $f(z) \in \gamma_{z}$ and $\left|f^{(r)}(z)-\Gamma^{(r)}(z)\right|<\varepsilon(z)$ for every $z \in \partial \Pi$ and every $0 \leq r \leq k$.

These results can also be seen as a one-sided version of Carleman type approximation. For example one has the following corollary.

Corollary 1.4. Let $a: \mathbb{R} \rightarrow \mathbb{C}$ and $\varepsilon: \mathbb{R} \rightarrow(0, \infty)$ be smooth functions on the real line. Then there exists a smooth up to the boundary holomorphic function $f$ on $\Pi$ such that $|f(z)-a(z)|=\varepsilon(z)$ for every $z \in \mathbb{R}$.

There are natural generalizations of previous results in the context of bordered Riemann surfaces. See $[3,6,7,9,10,11,20]$ for results which give sufficient conditions for the existence of solutions of the nonlinear Riemann-Hilbert problem on a finitely connected planar domains or more general bordered Riemann surfaces and which are smooth up to the whole boundary of the surface. On the other hand, if just one boundary point is removed, we always have a solution.

THEOREM 1.5. Let $\Sigma$ be a bordered Riemann surface with real analytic boundary, let $p_{0} \in \partial \Sigma$ and let $\left\{\gamma_{z}\right\}_{z \in \partial \Sigma \backslash\left\{p_{0}\right\}}$ be a smooth family of smooth Jordan curves in $\mathbb{C}$. Then there exists a smooth function $f$ on $\bar{\Sigma} \backslash\left\{p_{0}\right\}$, holomorphic on $\Sigma$, such that $f(z) \in \gamma_{z}$ for every $z \in \partial \Sigma \backslash\left\{p_{0}\right\}$.

Similarly as before we also have a version with approximation. 
THEOREM 1.6. Let $\left\{\gamma_{z}\right\}_{z \in \partial \Sigma \backslash\left\{p_{0}\right\}}$ be a smooth family of smooth Jordan curves in $\mathbb{C}$. Let $\Gamma: \partial \Sigma \backslash\left\{p_{0}\right\} \rightarrow \mathbb{C}$ be a $C^{k}(k \in \mathbb{N} \cup\{0\})$ function such that $\Gamma(z) \in \gamma_{z}$ for every $z \in \partial \Sigma \backslash\left\{p_{0}\right\}$ and let $\varepsilon: \partial \Sigma \backslash\left\{p_{0}\right\} \rightarrow(0, \infty)$ be a continuous positive function. Then there exists a holomorphic function $f$ on $\Sigma$, smooth up to $\partial \Sigma \backslash\left\{p_{0}\right\}$, such that $f(z) \in \gamma_{z}$ and $\left|f^{(r)}(z)-\Gamma^{(r)}(z)\right|<\varepsilon(z)$ for every $z \in \partial \Sigma \backslash\left\{p_{0}\right\}$ and every $0 \leq r \leq k$.

Results presented and proved in the last section are natural generalizations of the results proved in the first three sections. Formally it would be enough to present and prove just those results and skip some of the results in the first three sections. However, besides the fact that the first three sections of the paper could not be omitted completely and that some of the material would have to be presented anyway, we believe that the upper half-plane case is the most natural object to start our presentation with.

2. Some lemmas. In this section we prove some lemmas we need in the proof of the main results.

LEMMA 2.1. Let $a>0$ be a positive real number and let

$$
K=\{z \in \mathbb{C} ;|\operatorname{Re}(z)| \leq 3 a, 0 \leq \operatorname{Im}(z) \leq 3 a\} .
$$

Let $k \in \mathbb{N}$ and

$$
f: K \cup \mathbb{R} \longrightarrow \mathbb{C}
$$

a continuous function such that

a) $f: \mathbb{R} \longrightarrow \mathbb{C}$ is a $C^{k}$ function whose support is contained in $[-7 a, 7 a]$,

b) $f$ is holomorphic on the interior of $K$.

Then there exists a sequence of entire functions $\left\{p_{n}\right\}_{n \in \mathbb{N}}$ which

i) in the $C^{k}$ sense on $[-6 a, 6 a] \subset \mathbb{R}$ converges to $f$,

ii) uniformly on

$$
Q=\{z \in \mathbb{C} ;|\operatorname{Re}(z)| \leq a, 0 \leq \operatorname{Im}(z) \leq a\} .
$$

converges to $f$.

Lemma 2.1 is stated and proved in the form we can use it in our construction, however, its statement is far from being the most general approximation result one can get. See $[2,17,21,24]$ for more results. As in [2] convolution with the heat kernel will be used in our proof. Before we start with the proof of Lemma 2.1 we prove an elementary estimate.

Lemma 2.2. Let $a>0$. Let $z \in \mathbb{C}$ be such that $|\operatorname{Re}(z)| \leq a$ and $0 \leq \operatorname{Im}(z) \leq a$. Let $t>0$ and let $\xi \in \mathbb{C}$ be such that $|\operatorname{Re}(\xi)| \geq 3 a$ and $0 \leq \operatorname{Im}(\xi) \leq \operatorname{Im}(z)$. Then

$$
\left|e^{-\frac{(z-\xi)^{2}}{4 t}}\right| \leq e^{-\frac{3 a^{2}}{4 t}}
$$

Proof. We start with

$$
\left|e^{-\frac{(z-\xi)^{2}}{4 t}}\right|=e^{-\operatorname{Re}\left(\frac{(z-\xi)^{2}}{4 t}\right)}=e^{\frac{-(\operatorname{Re}(z)-\operatorname{Re}(\xi))^{2}+(\operatorname{Im}(z)-\operatorname{Im}(\xi))^{2}}{4 t}} .
$$

Since $0 \leq \operatorname{Im}(\xi) \leq \operatorname{Im}(z)$ and $0 \leq \operatorname{Im}(z) \leq a$, we have $(\operatorname{Im}(z)-\operatorname{Im}(\xi))^{2} \leq(\operatorname{Im}(z))^{2} \leq$ $a^{2}$. Also, because $|\operatorname{Re}(\xi)| \geq 3 a$ and $|\operatorname{Re}(z)| \leq a$, we have $(\operatorname{Re}(z)-\operatorname{Re}(\bar{\xi}))^{2} \geq(2 a)^{2}$. Thus

$$
\left|e^{-\frac{(z-\xi)^{2}}{4 t}}\right| \leq e^{\frac{a^{2}-4 a^{2}}{4 t}}=e^{-\frac{3 a^{2}}{4 t}} .
$$


Proof. (Lemma 2.1) For $t>0$ we define

$$
p_{t}(z)=\frac{1}{\sqrt{4 \pi t}} \int_{-\infty}^{\infty} e^{-\frac{(z-\xi)^{2}}{4 t}} f(\xi) d \xi
$$

Then $p_{t}$ is an entire function and

$$
p_{t}(z)=\frac{1}{\sqrt{\pi}} \int_{-\infty}^{\infty} e^{-\xi^{2}} f(z-\sqrt{4 t} \xi) d \xi
$$

for $z \in \mathbb{R}$. It is a classical result (see, for example, [12]) that $p_{t}$ converges to $f$ in $C^{k}$ sense on $[-6 a, 6 a]$.

Let $z_{0} \in Q$ and let

$$
K_{z_{0}}=\left\{\xi \in \mathbb{C} ;|\operatorname{Re}(\xi)| \leq 3 a, 0 \leq \operatorname{Im}(\xi) \leq \operatorname{Im}\left(z_{0}\right)\right\} .
$$

Since $f$ is holomorphic in the interior of $K$ we get by Cauchy formula

$$
\frac{1}{\sqrt{4 \pi t}} \int_{\partial K_{z_{0}}} e^{-\frac{\left(z_{0}-\xi\right)^{2}}{4 t}} f(\xi) d \xi=0 .
$$

From Lemma 2.2 we have an estimate on the vertical edges $I_{-}=\{\xi \in \mathbb{C} ; \operatorname{Re}(\xi)=$ $\left.-3 a, 0 \leq \operatorname{Im}(\xi) \leq \operatorname{Im}\left(z_{0}\right)\right\}$ and $I_{+}=\left\{\xi \in \mathbb{C} ; \operatorname{Re}(\xi)=3 a, 0 \leq \operatorname{Im}(\xi) \leq \operatorname{Im}\left(z_{0}\right)\right\}$ of the rectangle $K_{z_{0}}$

$$
\left|e^{-\frac{\left(z_{0}-\xi\right)^{2}}{4 t}}\right| \leq e^{-\frac{3 a^{2}}{4 t}}
$$

Since $f$ is bounded, the limits

$$
\lim _{t \downarrow 0} \frac{1}{\sqrt{4 \pi t}} \int_{I_{-}} e^{-\frac{\left(z_{0}-\xi\right)^{2}}{4 t}} f(\xi) d \xi=\lim _{t \downarrow 0} \frac{1}{\sqrt{4 \pi t}} \int_{I_{+}} e^{-\frac{\left(z_{0}-\xi\right)^{2}}{4 t}} f(\xi) d \xi=0
$$

exist and are both equal to 0. Similary we get from Lemma 2.2 and the assumption on the support of $f$ that

$$
\lim _{t \downarrow 0} \frac{1}{\sqrt{4 \pi t}} \int_{3 a}^{\infty} e^{-\frac{\left(z_{0}-\xi\right)^{2}}{4 t}} f(\xi) d \xi=\lim _{t \downarrow 0} \frac{1}{\sqrt{4 \pi t}} \int_{-\infty}^{-3 a} e^{-\frac{\left(z_{0}-\xi\right)^{2}}{4 t}} f(\xi) d \xi=0 .
$$

By the classical result [12] we have

$$
f\left(z_{0}\right)=\lim _{t \downarrow 0} \frac{1}{\sqrt{4 \pi t}} \int_{-3 a}^{3 a} e^{-\frac{\left(\operatorname{Re}\left(z_{0}\right)-\xi\right)^{2}}{4 t}} f\left(\xi+i \operatorname{Im}\left(z_{0}\right)\right) d \xi
$$

and the limit is uniform for $z_{0} \in Q$.

Therefore the limits

$$
\lim _{t \downarrow 0} p_{t}\left(z_{0}\right)=\lim _{t \downarrow 0} \frac{1}{\sqrt{4 \pi t}} \int_{-\infty}^{\infty} e^{-\frac{\left(z_{0}-\xi\right)^{2}}{4 t}} f(\xi) d \xi=\lim _{t \downarrow 0} \frac{1}{\sqrt{4 \pi t}} \int_{-3 a}^{3 a} e^{-\frac{\left(z_{0}-\xi\right)^{2}}{4 t}} f(\xi) d \xi
$$

exist and are equal to

$$
\lim _{t \downarrow 0} \frac{1}{\sqrt{4 \pi t}} \int_{-3 a}^{3 a} e^{-\frac{\left(\operatorname{Re}\left(z_{0}\right)-\xi\right)^{2}}{4 t}} f\left(\xi+i \operatorname{Im}\left(z_{0}\right)\right) d \xi=f\left(z_{0}\right) .
$$


Since all the estimates and limits we made are uniform with respect to $z_{0} \in Q$, we see that $p_{t}$ converges uniformly on $Q$ to $f$ as $t \downarrow 0$.

We also need solutions of the linear Riemann-Hilbert problem. Let $A(\Delta)$ denote the space of all continuous functions on $\bar{\Delta}$ which are holomorphic on $\Delta$. Let $k \in \mathbb{N} \cup\{0\}$ and $0<\alpha<1$. By $A^{k, \alpha}(\Delta)$ we will denote the Banach space $C^{k, \alpha}(\Delta) \cap A(\Delta)$ of all holomorphic functions on $\Delta$ which are of Hölder class $C^{k, \alpha}$ on $\Delta$.

Lemma 2.3. Let $\Delta \subseteq \mathbb{C}$ be the open unit disk centered at 0 . Let $I$ be a proper closed arc on $\partial \Delta$ and let $\omega: I \rightarrow \mathbb{C} \backslash\{0\}$ be a $C^{k, \alpha}$ function. Then the mapping

$$
\Phi: A^{k, \alpha}(\Delta) \longrightarrow C_{\mathbb{R}}^{k, \alpha}(I)
$$

defined as

$$
\Phi(f)=\operatorname{Re}(\bar{\omega} f)
$$

is a bounded surjective linear operator with a bounded right inverse

$$
B: C_{\mathbb{R}}^{k, \alpha}(I) \longrightarrow A^{k, \alpha}(\Delta) \text {. }
$$

In addition, our construction of the right inverse operator is made locally continuous, that is, if a sequence of nonzero functions $\left\{\omega_{n}\right\}_{n \in \mathbb{N}}$ on I in the $C^{k, \alpha}$ sense converges to $\omega$, then there are right inverses $B_{n}$ of the corresponding linear operators so that the sequence $\left\{B_{n}\right\}_{n \in \mathbb{N}}$ converges to $B$ in the operator norm.

REMARK 2.4. By $C_{\mathbb{R}}^{k, \alpha}(I)$ we denote the Banach space of all real $k$-times differentiable functions on $I$ whose $k$-th derivative is Hölder continuous of order $\alpha$.

Proof. It is obvious that $\Phi$ is a well defined bounded linear operator such that

$$
\|\Phi\| \leq\|\omega\|_{k, \alpha} .
$$

To show that it is surjective and that there is an appropriate right inverse we proceed as follows. Let

$$
H: C_{\mathbb{R}}^{k, \alpha}(\partial \Delta) \rightarrow C_{\mathbb{R}}^{k, \alpha}(\partial \Delta)
$$

denote the standard Hilbert transform and let $E$ be an extension operator on $I$, that is, a bounded linear operator

$$
E: C_{\mathbb{R}}^{k, \alpha}(I) \rightarrow C_{\mathbb{R}}^{k, \alpha}(\partial \Delta)
$$

such that

$$
\left.E(u)\right|_{I}=u
$$

for every $u \in C_{\mathbb{R}}^{k, \alpha}(I),[15]$.

Since $\omega$ is nonzero on $I$, there exists a well-defined logarithm of $\bar{\omega}$ on $I$

$$
\bar{\omega}=e^{b}
$$

where $b: I \rightarrow \mathbb{C}$ is of class $C^{k, \alpha}$. Let $E(b)=c+i d$, where $c$ and $d$ are real $C^{k, \alpha}$ functions on $\partial \Delta$. Then we define

$$
B(u)=e^{-i(d+i H d)}\left[e^{-(c+H d)} E(u)+i H\left(e^{-(c+H d)} E(u)\right)\right] .
$$


A simple computation

$$
\begin{gathered}
(\Phi \circ B)(u)=\operatorname{Re}(\bar{\omega} B(u))= \\
=\operatorname{Re}\left(e^{c+i d} e^{-i(d+i H d)}\left[e^{-(c+H d)} E(u)+i H\left(e^{-(c+H d)} E(u)\right)\right]\right)= \\
=\operatorname{Re}\left(E(u)+i e^{c+H d} H\left(e^{-(c+H d)} E(u)\right)\right)=E(u)=u
\end{gathered}
$$

shows that $\Phi \circ B$ is the identity on $C_{\mathbb{R}}^{k, \alpha}(I)$.

Let $\left\{\omega_{n}\right\}_{n \in \mathbb{N}}$ be a sequence of nonzero functions on $I$ which in the $C^{k, \alpha}$ sense converges to $\omega$. The corresponding logarithms $b_{n}$ of $\omega_{n}$ can be chosen so that the sequence $\left\{b_{n}\right\}_{n \in \mathbb{N}}$ in the $C^{k, \alpha}$ sense on $I$ converges to $b$. Thus the sequence of right inverses $\left\{B_{n}\right\}_{n \in \mathbb{N}}$ constructed as above in the operator norm converges to $B$.

3. Proofs of results on the open upper half plane. We will construct a solution of the Riemann-Hilbert problem on the upper half-plane inductively. Let $\rho \in C^{\infty}(\partial \Pi \times \mathbb{C})$ be a defining function for the family of Jordan curves $\left\{\gamma_{z}\right\}_{z \in \partial \Pi \text { and }}$ let $\Omega$ be a smoothly bounded convex domain in the upper half-plane such that its closure $\bar{\Omega}$ contains the rectangle

$$
R=\{z \in \mathbb{C} ;|\operatorname{Re}(z)| \leq 3,0 \leq \operatorname{Im}(z) \leq 3\}
$$

and that $\bar{\Omega} \cap \partial \Pi=[-3,3]$.

Proposition 3.1. Let $k \in \mathbb{N} \cup\{0\}$ and $\alpha \in(0,1)$. Let $a>0$ and

$$
f: a \Omega \longrightarrow \mathbb{C}
$$

an $A^{k, \alpha}(a \Omega)$ holomorphic function such that

$$
\rho(z, f(z))=0
$$

for every $z \in[-3 a, 3 a]$. Let $\varepsilon_{0}>0$. Then there exists a holomorphic function $g \in A^{k, \alpha}(2 a \Omega)$ such that

$$
\rho(z, g(z))=0
$$

for every $z \in[-6 a, 6 a]$ and $\|g-f\|_{\frac{a}{3} R}<\varepsilon_{0}$.

In the proof of Proposition 3.1 we will use the following result due to Begehr and Efendiev [3] based on the Newton's iteration method.

Theorem 3.2 (Begehr-Efendiev). Let $X$ and $Y$ be Banach spaces. Let $A: X \rightarrow$ $Y$ be a continuous mapping, Fréchet differentiable in a neighbourhood of $x_{0} \in X$. Assume that $(D A)\left(x_{0}\right)$ has a bounded right inverse $B\left(x_{0}\right)$ with the norm $\omega_{1}>0$. Also, let $\omega_{2}>0$ be such that the derivative $(D A)(x)$ satisfies a Lipschitz condition

$$
\left\|(D A)\left(x_{1}\right)-(D A)\left(x_{2}\right)\right\|<\omega_{2}\left\|x_{1}-x_{2}\right\|
$$

for $x_{1}, x_{2}$ in a neighbourhood of $x_{0}$. Finally, let $\omega_{3}>0$ be such that

$$
4 \omega_{1}\left(\omega_{1}+1\right)\left(\omega_{2}+1\right) \omega_{3}<1
$$


Then for every $y \in Y$ such that $\left\|A\left(x_{0}\right)-y\right\|<\omega_{3}$ there exists a solution of the equation $A(x)=y$.

It follows from the proof of Theorem 3.2 that solution of the equation $A(x)=y$, which one gets using Newton's iteration method, is close to the initial data $x_{0}$. The distance is, under the assumptions of the theorem, bounded by the product $2 \omega_{1} \omega_{3}$.

Proof. (Proposition 3.1) Recall that by a theorem of Cirka [8], $f$ is smooth on $[-3 a, 3 a]$. We extend $f$ to a smooth function on $\partial \Pi$ with the support in $[-7 a, 7 a]$ and such that

$$
\rho(z, f(z))=0 \quad z \in[-6 a, 6 a]
$$

Remark 3.3. Let $\Gamma: \partial \Pi \rightarrow \mathbb{C}$ be a $C^{k}(k \in \mathbb{N} \cup\{0\})$ function such that $\Gamma(z) \in \gamma_{z}$ for every $z \in \partial \Pi$ and let $\varepsilon: \partial \Pi \rightarrow(0, \infty)$ be a continuous positive function. If $\left|f^{(r)}(z)-\Gamma^{(r)}(z)\right|<\varepsilon(z)$ on $[-3 a, 3 a]$ and every $0 \leq r \leq k$, we can extend $f$ in such a way that $\left|f^{(r)}(z)-\Gamma^{(r)}(z)\right|<\varepsilon(z)$ on $[-6 a, 6 a]$ and every $0 \leq r \leq k$.

By Lemma 2.1 there exists a sequence of entire functions $\left\{p_{n}\right\}_{n \in \mathbb{N}}$ which

i) in the $C^{k+1}$ sense on $[-6 a, 6 a] \subset \partial \Pi$ converges to $f$,

ii) uniformly on

$$
Q=\{z \in \mathbb{C} ;|\operatorname{Re}(z)| \leq a, 0 \leq \operatorname{Im}(z) \leq a\} .
$$

converges to $f$.

Let $X=A^{k, \alpha}(2 a \Omega)$ and let $Y=C_{\mathbb{R}}^{k, \alpha}([-6 a, 6 a])$. Let $A: X \rightarrow Y$ be a nonlinear operator defined as $A(\varphi)(z)=\rho(z, \varphi(z))$ for every $\varphi \in X$ and every $z \in[-6 a, 6 a]$. From [16] it follows that $A$ is a $C^{2}$ smooth mapping with the derivative

$$
\varphi \longmapsto 2 \operatorname{Re}\left(\partial_{w} \rho\left(z, \varphi_{0}(z)\right) \varphi(z)\right) .
$$

at point $\varphi_{0} \in X$. From the smoothness of the defining function $\rho$ we have

$$
\lim _{n \rightarrow \infty} \rho\left(z, p_{n}(z)\right)=0
$$

in the $C^{k, \alpha}$ sense on $[-6 a, 6 a]$. Hence $\lim _{n \rightarrow \infty} A\left(p_{n}\right)=0$ in $Y$. Also, for $n$ large enough is the second derivative of the operator $A$ at the point $p_{n}$ in norm close to the bilinear mapping

$$
(\varphi, \psi) \in X \times X \longmapsto 2 \operatorname{Re}\left(\partial_{w w}^{2} \rho(z, f(z)) \varphi(z) \psi(z)+\partial_{w \bar{w}}^{2} \rho(z, f(z)) \varphi(z) \overline{\psi(z)}\right) \in Y .
$$

Finally, the right inverse $B_{n}$ to the derivative $(D A)\left(p_{n}\right)$ constructed in Lemma 2.3 is in norm close to the right inverse of the operator

$$
\varphi \longmapsto \operatorname{Re}\left(\partial_{w} \rho(z, f(z)) \varphi(z)\right) .
$$

By Theorem 3.2 we conclude that there is a holomorphic function $g \in A^{k, \alpha}(2 a \Omega)$ such that $A(g)(z)=\rho(z, g(z))=0$ for every $z \in[-6 a, 6 a]$,

$$
\left|g^{(r)}(z)-f^{(r)}(z)\right|<\varepsilon(z)
$$

for every $z \in[-6 a, 6 a], 0 \leq r \leq k$, and $\|g-f\|_{\frac{a}{3} R}<\varepsilon_{0}$. 
Proof. (Theorem 1.1) The proof of Theorem 1.1 is clear. For $n \in \mathbb{N}$ let $\Omega_{n}=2^{n} \Omega$, $R_{n}=2^{n} R, I_{n}=2^{n}[-3,3]$ and $\varepsilon_{n}=\frac{1}{2^{n}}$.

We let $f_{1} \in A^{k, \alpha}\left(\Omega_{1}\right)$ be any function for which $\rho\left(z, f_{1}(z)\right)=0$ on $I_{1}$. One can get such function using Lemma 2.1 and results from [13, 23].

REMARK 3.4. If, in addition, we also need some approximation of a given function $\Gamma$ we proceed as follows: we first approximate $\Gamma$ in the $C^{k}$ sense on $\partial \Pi$ by a smooth function $\widetilde{\Gamma}$ for which $\rho(z, \widetilde{\Gamma}(z))=0$ for every $z \in \partial \Pi$. Then we get, using Lemma 2.1, a sequence $\left\{p_{m}\right\}_{m \in \mathbb{N}}$ of entire functions which in the $C^{k+1}$ sense converges to $\widetilde{\Gamma}$ on $I_{1}$. Lastly we use Theorem 3.2 as in the proof of Proposition 3.1 to get a function $f_{1} \in A^{k, \alpha}\left(\Omega_{1}\right)$ so that $\rho\left(z, f_{1}(z)\right)=0$ and $\left|\widetilde{\Gamma}^{(r)}(z)-f_{1}^{(r)}(z)\right|<\varepsilon(z)$ for every $z \in I_{1}$ and $0 \leq r \leq k$.

Now we use Proposition 3.1 inductively to construct a sequence $\left\{f_{n}\right\}_{n \in \mathbb{N}}$ of functions $f_{n} \in A^{k, \alpha}\left(\Omega_{n}\right)$ such that

1. $\rho\left(z, f_{n}(z)\right)=0$ for $z \in I_{n}$,

2. $\left\|f_{n}-f_{n+1}\right\|_{\frac{1}{3} R_{n}}<\varepsilon_{n}$.

The second condition implies that the sequence $\left\{f_{n}\right\}_{n \in \mathbb{N}}$ converges uniformly on compact subsets of $\bar{\Pi}$ to a continuous function $f: \bar{\Pi} \rightarrow \mathbb{C}$ which is holomorphic on $\Pi$. The first condition implies that $f$ is a solution of the Riemann-Hilbert problem for

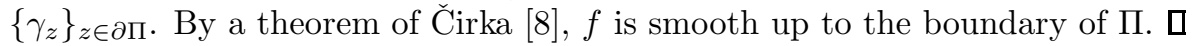

The same arguments, with the necessary modifications which we have already indicated along the proof, actually show a stronger result.

THEOREM 3.5. Let $\left\{\gamma_{z}\right\}_{z \in \partial \Pi}$ be a smooth family of smooth Jordan curves in $\mathbb{C}$. Let $\Gamma: \partial \Pi \rightarrow \mathbb{C}$ be a $C^{k}(k \in \mathbb{N} \cup\{0\})$ function such that $\Gamma(z) \in \gamma_{z}$ for every $z \in \partial \Pi$ and let $\varepsilon: \partial \Pi \rightarrow(0, \infty)$ be a positive continuous function. Then there exists a smooth up to the boundary holomorphic function $f$ on $\Pi$ such that $f(z) \in \gamma_{z}$ and $\left|f^{(r)}(z)-\Gamma^{(r)}(z)\right|<\varepsilon(z)$ for every $z \in \partial \Pi$ and every $0 \leq r \leq k$.

REMARK 3.6. Function $f$ could also be constructed to agree with $\Gamma$ up to order $k$ on a prescribed discrete set of points on $\partial \Pi$.

4. Bordered Riemann surfaces. Let $\Sigma$ be a bordered Riemann surface with real analytic boundary and let $p_{0} \in \partial \Sigma$. Let $\left\{\gamma_{z}\right\}_{z \in \partial \Sigma \backslash\left\{p_{0}\right\}}$ be a smooth family of smooth Jordan curves in $\mathbb{C}$ defined by a defining function $\rho \in C^{\infty}\left(\left(\partial \Sigma \backslash\left\{p_{0}\right\}\right) \times \mathbb{C}\right)$. As a natural generalization of Theorem 1.2 we have the following theorem.

THEOREM 4.1. Let $\left\{\gamma_{z}\right\}_{z \in \partial \Sigma \backslash\left\{p_{0}\right\}}$ be a smooth family of smooth Jordan curves in $\mathbb{C}$. Then there exists a smooth function $f$ on $\bar{\Sigma} \backslash\left\{p_{0}\right\}$, holomorphic on $\Sigma$, such that $f(z) \in \gamma_{z}$ for every $z \in \partial \Sigma \backslash\left\{p_{0}\right\}$.

Similarly as before we need solutions of the linear Riemann-Hilbert problem. Let $k \in \mathbb{N} \cup\{0\}$ and $0<\alpha<1$.

Lemma 4.2. Let $\Sigma$ be a bordered Riemann surface with genus $g$ and $m$ real analytic boundary components. Let $I$ be a proper closed arc of $\partial \Sigma$ and let $\omega: I \rightarrow$ $\mathbb{C} \backslash\{0\}$ be a $C^{k, \alpha}$ function. Then the mapping

$$
\Phi: A^{k, \alpha}(\Sigma) \longrightarrow C_{\mathbb{R}}^{k, \alpha}(I)
$$

defined as

$$
\Phi(f)=\operatorname{Re}(\bar{\omega} f)
$$


is a bounded surjective linear operator with a bounded right inverse

$$
B: C_{\mathbb{R}}^{k, \alpha}(I) \longrightarrow A^{k, \alpha}(\Sigma) \text {. }
$$

In addition, our construction of the right inverse operator can be made locally continuous, that is, if a sequence of nonzero functions $\left\{\omega_{n}\right\}_{n \in \mathbb{N}}$ on $I$ in the $C^{k, \alpha}$ sense converges to $\omega$, then there are right inverses $B_{n}$ of the corresponding linear operators so that the sequence $\left\{B_{n}\right\}_{n \in \mathbb{N}}$ converges to $B$ in the operator norm.

Proof. Mapping $\Phi$ is a well defined bounded linear operator. Using an extension operator $E$ [15] we extend $\omega$ to a nonzero $C^{k, \alpha}$ function on $\partial \Sigma$. Let $W(\omega)$ denote the winding number of (the extended function) $\omega$ along $\partial \Sigma$, where $\partial \Sigma$ is oriented coherently with the natural orientation of $\Sigma$ as a Riemann surface. Let $J$ be a closed subarc of $\partial \Sigma \backslash I$ and let $\mu: \partial \Sigma \rightarrow \mathbb{C} \backslash\{0\}$ be a smooth nowhere zero function such that $\mu=1$ on $\partial \Sigma \backslash J$, and such that the winding number $W(\mu)$ of $\mu$ along $\partial \Sigma$ is

$$
W(\mu)=-W(\omega)+2 g+m-1 .
$$

Then $z \mapsto \mu(z) \omega(z)$ is a well defined nonzero $C^{k, \alpha}$ function on $\partial \Sigma$ such that $W(\mu \omega)=$ $2 g+m-1$. By [19] we have that the mapping

$$
\widetilde{\Phi}: f \longmapsto \operatorname{Re}(\overline{(\mu \omega)} f)
$$

from $A^{k, \alpha}(\Sigma)$ into $C_{\mathbb{R}}^{k, \alpha}(\partial \Sigma)$ is surjective with $2 W(\mu \omega)-(2 g+m-2)=2 g+m$ dimensional kernel and hence there is a well defined bounded right inverse operator

$$
\widetilde{B}: C_{\mathbb{R}}^{k, \alpha}(\partial \Sigma) \longrightarrow A^{k, \alpha}(\Sigma)
$$

Finally we define $B=\widetilde{B} \circ E$.

Let $\left\{\omega_{n}\right\}_{n \in \mathbb{N}}$ be a sequence of nonzero functions on $I$ which in the $C^{k, \alpha}$ sense converges to $\omega$. Without loss of generality we may assume that functions $\omega_{n}(n \in \mathbb{N})$ are already defined and nonzero on $\partial \Sigma$. Each function $\omega_{n}(n \in \mathbb{N})$ defines a bounded linear operator

$$
\widetilde{\Phi}_{n}: A^{k, \alpha}(\Sigma) \longrightarrow C_{\mathbb{R}}^{k, \alpha}(\partial \Sigma)
$$

defined as

$$
\widetilde{\Phi}_{n}: f \longmapsto \operatorname{Re}\left(\overline{\left(\mu \omega_{n}\right)} f\right) .
$$

Since $\widetilde{B}$ is a bounded right inverse of the operator $\widetilde{\Phi}$, its image is a closed subspace of $A^{k, \alpha}(\Sigma)$ such that

$$
A^{k, \alpha}(\Sigma)=\operatorname{Im}(\widetilde{B}) \oplus \operatorname{Ker}(\widetilde{\Phi})
$$

and $\widetilde{\Phi}: \operatorname{Im}(\widetilde{B}) \rightarrow C^{k, \alpha}(\partial \Sigma)$ is an isomorphism. Hence the same is true for the operators $\widetilde{\Phi}_{n}$. Finally, the operator $B_{n}(n \in \mathbb{N})$ is defined as the inverse of the operator

$$
\widetilde{\Phi}_{n}: \operatorname{Im}(\widetilde{B}) \longrightarrow C_{\mathbb{R}}^{k, \alpha}(\partial \Sigma)
$$

composed with $E$. 
REMARK 4.3. On an arbitrary bordered Riemann surface $\Sigma$ there is no canonical way to define the right inverse operators $B_{n}$ as it was done on the disc in Lemma 2.3.

Proof. (Theorem 4.1) Let $D$ be a smoothly bounded simply connected domain in $\Sigma$ such that $\bar{D} \cap \partial \Sigma$ is a closed arc which contains point $p_{0}$ in its interior. Also, let $q_{0} \in \partial D \cap \operatorname{Int}(\Sigma)$. Then there is a biholomorphic map $\psi$ from $D$ onto $\Pi$ that is smooth on $\bar{D} \backslash\left\{p_{0}\right\}$, which takes point $q_{0}$ to 0 , point $p_{0}$ to infinity and such that the preimage by $\psi$ of the half-lines $(-\infty,-1] \cup[1, \infty)$ belongs to the boundary $\partial \Sigma$.

Let $P(a) \subset \bar{\Sigma}(a>0)$ be the preimage by $\psi$ of the closed rectangle $\{z \in$ $\mathbb{C} ;|\operatorname{Re}(z)| \leq 3 a, 0 \leq \operatorname{Im}(z) \leq 3 a\}$ and let $\Omega_{n} \subseteq \Pi$ be as in the proof of Theorem 1.1. For $n \in \mathbb{N}$ we define smoothly bounded domains

$$
U_{n}=\psi^{-1}\left(\Omega_{n}\right) \cup(\Sigma \backslash D) .
$$

We will denote by $\partial^{\prime} U_{n}$ the part of the boundary of $U_{n}$ which belongs to the boundary of $\Sigma$, that is,

$$
\partial^{\prime} U_{n}=\partial U_{n} \cap \partial \Sigma
$$

Finally, let $\chi$ be a smooth function on $\bar{\Sigma}$ whose support is contained in $\bar{D} \backslash P\left(\frac{1}{3}\right)$ and which equals 1 on $\bar{D} \backslash P\left(\frac{1}{2}\right)$.

Proposition 4.4. Let $k \in \mathbb{N} \cup\{0\}$ and $\alpha \in(0,1)$. Let

$$
f_{n}: U_{n} \longrightarrow \mathbb{C}
$$

be a holomorphic function of class $C^{k, \alpha}$ such that

$$
\rho\left(z, f_{n}(z)\right)=0
$$

for every $z \in \partial^{\prime} U_{n}$. Let $\varepsilon_{0}>0$. Then there exists a holomorphic function $f_{n+1} \in$ $A^{k, \alpha}\left(U_{n+1}\right)$ such that

$$
\rho\left(z, f_{n+1}(z)\right)=0
$$

for every $z \in \partial^{\prime} U_{n+1}$ and $\left\|f_{n+1}-f_{n}\right\|_{P\left(\frac{1}{3} 2^{n}\right) \cup(\overline{\Sigma \backslash D})}<\varepsilon_{0}$.

Before we start with the proof of Proposition 4.4 we observe that the sequence of entire functions $\left\{p_{n}\right\}_{n \in \mathbb{N}}$ constructed in Lemma 2.1 converges to $f$ in the $C^{k}$ sense on $Q$.

Proof. The proof of Proposition 4.4 is similar to the proof of Proposition 3.1. However, since we are working on an arbitrary bordered Riemann surface, we need to solve a $\bar{\partial}$-problem to get function $f_{n+1}$.

Recall that by a theorem of Cirka [8], $f_{n}$ is smooth on $\partial^{\prime} U_{n}$. We extend $f_{n}$ to a smooth function on $\partial \Sigma$ with support in a neighbourhood of $\partial^{\prime} U_{n+1}$ and such that $\rho\left(z, f_{n}(z)\right)=0$ on $\partial^{\prime} U_{n+1}$. If necessary, we can extend $f_{n}$ so that it approximates a given function $\Gamma$ with values in $\left\{\gamma_{z}\right\}_{z \in \partial \Sigma \backslash\left\{p_{0}\right\}}$. By Lemma 2.1 there exists a sequence of holomorphic functions $\left\{p_{m}\right\}_{m \in \mathbb{N}}$ on $D$, smooth up to $\partial D \backslash\left\{p_{0}\right\}$, such that

i) in the $C^{k+1}$ sense on $\partial \psi^{-1}\left(\Omega_{n+1}\right) \cap \partial \Sigma$ converges to the extended function $f_{n}$

ii) uniformly on $P\left(\frac{1}{3} 2^{n}\right)$ converges to $f_{n}$. 
We define

$$
\widetilde{f}_{m, n}=\chi p_{m}+(1-\chi) f_{n} .
$$

Then $\widetilde{f}_{m, n}$ converges in the $C^{k+1}$ sense on $\partial^{\prime} U_{n+1}$ to the extended function $f_{n}$ and

$$
\bar{\partial} \widetilde{f}_{m, n}=\bar{\partial} \chi\left(p_{m}-f_{n}\right)
$$

converges in the $C^{k}$ norm on $\Sigma$ to 0 . Solving $\bar{\partial}$ equation on $\Sigma$ we get a holomorphic function $h_{m, n}$ on $\Sigma$ which is in the $C^{k, \alpha}$ sense close to $\widetilde{f}_{m, n}$ on $\partial^{\prime} U_{n+1}$ and uniformly close to $f_{n}$ on $P\left(\frac{1}{3} 2^{n}\right) \cup(\overline{\Sigma \backslash D})$. Using Lemma 4.2 and Theorem 3.2 as before we show that there exists a holomorphic function $f_{n+1}$ from $A^{k, \alpha}\left(U_{n+1}\right)$ which solves the Riemann-Hilbert problem on $\partial^{\prime} U_{n+1}$ and which is uniformly arbitrarily close to $f_{n}$ on $P\left(\frac{1}{3} 2^{n}\right) \cup(\overline{\Sigma \backslash D})$.

To complete the proof of Theorem 4.1 we need to construct function $f_{1}$. The rest is done by inductive procedure like in the proof of Theorem 1.1.

Let $\Gamma: \partial \Sigma \backslash\left\{p_{0}\right\} \rightarrow \mathbb{C}$ be a $C^{k}$ function such that $\Gamma(z) \in \gamma_{z}$ for every $z \in \partial \Sigma \backslash\left\{p_{0}\right\}$. We approximate $\Gamma$ in the $C^{k}$ sense on $\partial \Sigma \backslash\left\{p_{0}\right\}$ by a smooth function $\widetilde{\Gamma}$ for which $\rho(z, \widetilde{\Gamma}(z))=0$ for every $z \in \partial \Sigma \backslash\left\{p_{0}\right\}$.

Without loss of generality we may assume that $\bar{\Sigma}$ is a closed subset of an open Riemann surface $\widetilde{\Sigma}$ with the same genus and the same number of boundary components as $\Sigma$. Each boundary component $K$ of $\Sigma$ has a neighbourhood in $\widetilde{\Sigma}$ which is conformally equivalent to some annulus

$$
A_{a}=\left\{\xi \in \mathbb{C} ; e^{-a}<|\xi|<e^{a}\right\} \quad(a>0)
$$

and such that $K$ is mapped by the conformal equivalence to the unit circle $|\xi|=1$. Using the mapping $e^{-i \xi}$ which takes the strip

$$
S_{a}=\{\xi \in \mathbb{C} ;-a<\operatorname{Im}(\xi)<a\}
$$

onto $A_{a}$ and the convolution with the heat kernel, we conclude that there is a sequence of holomorphic functions on a neighbourhood of $K$ in $\widetilde{\Sigma}$ which in the $C^{k+1}$ sense converges to $\widetilde{\Gamma}$ on $K$. Observe that the convolution of a periodic function on $\mathbb{R}$ with the heat kernel gives a periodic entire function with the same period. For the boundary component $K_{0}$ of $\Sigma$ which contains the point $p_{0}$ the conclusion of approximation is done only in a neighbourhood of $K_{0} \cap \partial^{\prime} U_{1}$ in $\widetilde{\Sigma}$. By a generalized Runge's theorem $[4,5,18]$ then there exists a sequence $\left\{q_{n}\right\}_{n \in \mathbb{N}}$ of holomorphic functions on $\widetilde{\Sigma}$ which in the $C^{k+1}$ sense on $\partial^{\prime} U_{1}$ converges to $\widetilde{\Gamma}$.

We use Theorem 3.2 and Lemma 4.2 again to show that there exists a holomorphic function $f_{1}$ on $U_{1}$ of class $C^{k, \alpha}$ which solves the Riemann-Hilbert problem on $\partial^{\prime} U_{1}$ and which is on $\partial^{\prime} U_{1}$ in the $C^{k}$ sense close to $\widetilde{\Gamma}$ and hence also to $\Gamma$. $\square$

As before we also have a version with approximation.

THEOREM 4.5. Let $\left\{\gamma_{z}\right\}_{z \in \partial \Sigma \backslash\left\{p_{0}\right\}}$ be a smooth family of smooth Jordan curves in $\mathbb{C}$. Let $\Gamma: \partial \Sigma \backslash\left\{p_{0}\right\} \rightarrow \mathbb{C}$ be a $C^{k}(k \in \mathbb{N} \cup\{0\})$ function such that $\Gamma(z) \in \gamma_{z}$ for every $z \in \partial \Sigma \backslash\left\{p_{0}\right\}$ and let $\varepsilon: \partial \Sigma \backslash\left\{p_{0}\right\} \rightarrow(0, \infty)$ be a continuous positive function. Then there exists a holomorphic function $f$ on $\Sigma$, smooth up to $\partial \Sigma \backslash\left\{p_{0}\right\}$, such that $f(z) \in \gamma_{z}$ and $\left|f^{(r)}(z)-\Gamma^{(r)}(z)\right|<\varepsilon(z)$ for every $z \in \partial \Sigma \backslash\left\{p_{0}\right\}$ and every $0 \leq r \leq k$.

REMARK 4.6. Using the same technique and a result on the boundary regularity of analytic varieties [8], one can prove similar statements under the assumption that 
$\rho \in C^{k+1}\left(\left(\partial \Sigma \backslash\left\{p_{0}\right\}\right) \times \mathbb{C}\right)$ for some $k \geq 2$. In that case one gets solutions of the Riemann-Hilbert problem of Hölder class $C^{k, \alpha}$.

\section{REFERENCES}

[1] H. Alexander, Gromov's method and Bennequin's problem, Invent. Math., 125 (1996), pp. $135-148$.

[2] M. S. BaOuendi And F. Treves, A property of the functions and distributions annihilated by a locally integrable system of complex vector fields, Ann. of Math. (2), 113 (1981), pp. 387-421.

[3] H. Begehr And M. A. Efendiev, On the asymptotics of meromorphic solutions for nonlinear Riemann-Hilbert problems, Math. Proc. Cambridge Philos. Soc., 127 (1999), pp. 159-172.

[4] H. Behnke AND K. Stein, Entwicklung analytischer Funktionen auf Riemannschen Flächen, Math. Ann., 120 (1949), pp. 430-461.

[5] E. Bishop, Subalgebras of functions on a Riemann surface, Pacific J. Math., 8 (1958), pp. 2950 .

[6] M. ČERNE, Nonlinear Riemann-Hilbert problem for bordered Riemann surfaces, Amer. J. Math., 126 (2004), pp. 65-85.

[7] M. Černe and M. Flores, Generalized Ahlfors functions, Trans. Amer. Math. Soc., 359 (2007), pp. 671-686.

[8] E. M. ĊIRKA, Regularity of boundaries of analytic sets, (Russian) Math. Sb. (NS), 117 (1982), pp. 291-334.

[9] M. A. Efendiev and W. L. Wendland, Nonlinear Riemann-Hilbert problems for multiply connected domains, Nonlinear Anal., 27 (1996), pp. 37-58.

[10] M. A. Efendiev And W. L. Wendland, Nonlinear Riemann-Hilbert problems without transversality, Math. Nachr., 183 (1997), pp. 73-89.

[11] M. A. Efendiev and W. L. Wendland, Nonlinear Riemann-Hilbert problems for doubly connected domains and closed boundary data, Topol. Methods Nonlinear Anal., 17 (2001), pp. 111-124.

[12] G. B. Folland, Introduction to partial differential equations, Princeton University Press, Princeton, 1995.

[13] F. Forstnerič, Polynomial hulls of sets fibered over the circle, Indiana Univ. Math. J., 37 (1988), pp. 869-889.

[14] P. M. Gauthier and W. Hengartner, Uniform approximation on closed sets by functions analytic on a Riemann surface, Approximation theory (Proc. Conf. Inst. Math., Adam Mickiewicz Univ., Poznań, 1972), pp. 63-69. Reidel, Dordrecht, 1975.

[15] D. Gilbarg and N. S. Trudinger, Elliptic partial differential equations of second order, Grundlehren der Mathematischen Wissenschaften, Vol. 224, Springer, Berlin-New York, 1977.

[16] C. D. Hill and G. TAiani, Families of analytic discs in $\mathbb{C}^{n}$ with boundaries on prescribed CR submanifold, Ann. Scuola. Norm. Sup. Pisa, 5 (1978), pp. 327-380.

[17] W. Kaplan, Approximation by entire functions, Michigan Math. J., 3 (1955), pp. 43-52.

[18] L. K. Kodama, Boundary measures of analytic differentials and uniform approximation on a Riemann surface, Pacific J. Math., 15 (1965), pp. 1261-1277.

[19] W. Koppelman, The Riemann-Hilbert problem for finite Riemann surfaces, Comm. Pure Appl. Math., 12 (1959), pp. 13-35.

[20] F. G. Maksudov and M. A. Efendiev, The nonlinear Hilbert problem for a doubly connected domain (Russian), Dokl. Akad. Nauk SSSR, 290 (1986), pp. 789-791.

[21] S. Scheinberg, Uniform approximation by entire functions, J. Analyse Math., 29 (1976), pp. $16-18$.

[22] Z. Slodkowski, Polynomial hulls in $\mathbb{C}^{2}$ and quasicircles, Ann. Scuola Norm. Sup. Pisa, 16 (1989), pp. 367-391.

[23] A. I. ŠniRELman, The degree of a quasiruled mapping and a nonlinear Hilbert problem, (Russian) Mat. Sb., 18 (1972), pp. 373-396.

[24] J. Verdera, On $C^{m}$ rational approximation, Proc. Amer. Math. Soc., 97 (1986), pp. 621-625.

[25] E. Wegert, Nonlinear boundary value problems for holomorphic functions and singular integral equations, Mathematical Research, 65, Akademie-Verlag, Berlin, 1992. 